\title{
PULSED THZ INTERROGATION OF SOFI WITH KNIT LINES IN 2D
}

\author{
H. T. Banks ${ }^{1}$, N. L. Gibson ${ }^{1}$, and W. P. Winfree ${ }^{2}$ \\ ${ }^{1}$ Center for Research and Scientific Computation, Box 8205, \\ North Carolina State University, Raleigh, NC 27606- \\ 8205 \\ ${ }^{2}$ NASA Langley Research Center, MS 231, Hampton, VA \\ 23681-2199
}

\begin{abstract}
This paper examines the scattering effect of knit lines and voids in SOFI through simulations of $\mathrm{THz}$ interrogation at normal and non-normal angles of incidence and using focused and non-focused single-cycle plane waves. We model the electromagnetic field using the TE mode of the 2D Maxwell's equations reduced to a wave equation, which are then solved with a finite-element time-domain method. The knit lines are modeled by changing the dielectric constant.
\end{abstract}

Keywords: THz interrogation, SOFI, knit lines, FEM, time domain.

PACS: 41.20.Jb, 02.60.Cb, 02.70.Dh

\section{INTRODUCTION}

Pulsed $\mathrm{THz}$ frequency waves have been shown to be particularly useful for the detection of voids in the Sprayed on Foam Insulation (SOFI) used on the Space Shuttle's Thermal Protection System (TPS) [1]. However, the modeling of the propagation of a $\mathrm{THz}$ pulse inside of a material which exhibits heterogeneous micro-structures of sizes that are on the order of the wave length of the interrogating field is not straight-forward. Additionally, data on the dielectric properties of low density foam in the $\mathrm{THz}$ regime is rather sparse. Initial efforts to remedy this deficiency can be found in [2].

As discussed in [3], usual approaches to THz interrogation of SOFI generally employ signal processing techniques (for example, see [4]), which do not take advantage of much of the information contained in the reflected data signal. A physics based model may be able to more accurately describe defects [5].

Previous efforts $([5,6])$ investigated the application of polarization mechanisms to account for the attenuation of wave propagation in foam, but 
matching the simulations to experimental data has yet to be completely successful. While these models were expressed in only one spatial dimension, the 2D formulation in [3] allowed for non-normally incident angles and curved interfaces. Further, by allowing the dielectric constant to be piecewise-constant, layers of differing densities can be modeled explicitly.

SOFI is applied in layers, producing interfaces between layers that have increased density (called "knit lines") which scatter the interrogating waveform. As the knit lines are generally on the order of $.5 \mathrm{~mm}$ thick, they are frequently ignored in simulations. But, as there may be as many as $20 \mathrm{knit}$ lines in a typical $20 \mathrm{~cm}$ block of foam, the aggregate effect on the interrogating signal can be significant. This paper describes our efforts to understand and quantify the scattering mechanisms, as well as to minimize their effects by the use of focusing and/or altering the angle of incidence. We consider the scattering of a $\mathrm{THz}$ plane wave off of the knit lines, modeled as layers of increased density, and voids, modeled as pockets of no density, inside a block of low density polyurethane foam.

An outline of the paper is as follows. First we present the particular form of Maxwell's equations we use to model the 2D electromagnetic interrogation problem for foam. Then an experimentally based approach for estimating the material parameters for use in the system is described. We next briefly report on our numerical techniques for solving the resulting system. Finally we present simulations for cases in which the angle of incidence is altered, and the plane wave is focused to a point.

\section{A SIMPLIFIED MATHEMATICAL MODEL}

For the computational domain we take a rectangular region $0 \leq x \leq 0.1 \mathrm{~m}$ and $0 \leq y \leq 0.2 \mathrm{~m}$. Figure 1 shows a schematic region containing a material with knit lines (represented by dashed lines) $1 \mathrm{~mm}$ from each other, each parallel to an approaching plane wave, and perpendicular to the direction of propagation. The regions between knit lines consist of a low density material, and the elliptical pocket (void) is modeled as having zero density. The far right boundary $(y=0.2)$ is assumed to be metallic, and therefore supra-conducting, thus simulating the aluminum backing of the SOFI on the shuttle external tank. A vacuum is present to the left of the material in which the interrogating field will be generated. 


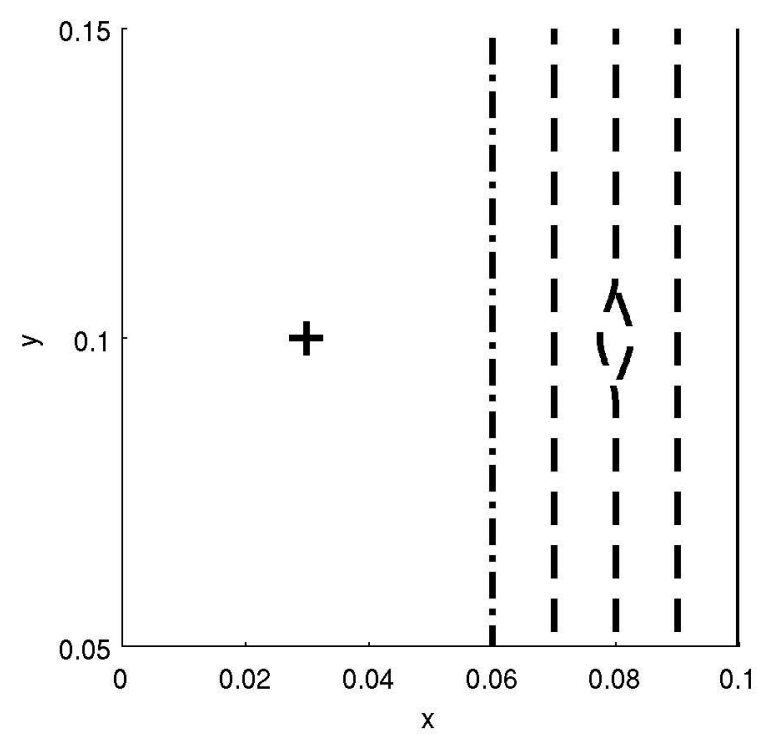

FIGURE 1: Schematic of a plane wave approaching a domain containing knit lines and an elliptical void. Dashed lines represent knit lines, dot-dash is foam/air interface. Elliptical pocket ( $5 \mathrm{~mm}$ wide) between knit lines is a void modeled by $n=1$. "+" marks the signal receiver. Back wall is perfect conductor.

We combine the TE mode of the two dimensional Maxwell's equations into one equation

$$
\epsilon(\vec{x}) \frac{\partial^{2} E}{\partial t}(t, \vec{x})+\nabla \cdot\left(\frac{1}{\mu(\vec{x})} \nabla E(t, \vec{x})\right)=-\frac{\partial J_{s}}{\partial t}(t, \vec{x}),
$$

where $\epsilon(\vec{x})$ and $\mu(\vec{x})$ are the spatially dependent dielectric permittivity and permeability, respectively. The corresponding speed of propagation is

$$
c(\vec{x})=\frac{c_{0}}{n(\vec{x})}=\sqrt{\frac{1}{\epsilon(\vec{x}) \mu(\vec{x})}},
$$

where $c_{0}$ is the speed in a vacuum.

For our source current, $J_{s}$, we want to simulate a windowed pulse, in this case a pulse that is allowed to oscillate for one half of one period and then is truncated. Although generators produce a curved, sometimes spherical wave, we assume the target is sufficiently far from the generator (approximately 6") so that the wave is essentially planar when it reaches our domain of interest. Thus, we want the pulse to originate at $x=0$, the beginning of 
our computation domain, which we model as a delta distribution centered at $x=0$. In order to have a smooth source we use a function of the form

$$
J_{s}(t, \vec{x})=\delta(x) e^{-\left(\left(t-t_{0}\right) / t_{0}\right)^{b}}
$$

where $t_{0}=t_{f} / 4$ when $t_{f}$ is the period of the interrogating pulse. For example, if the frequency is $f=.2 \mathrm{THz}$, then $t_{f}=1 / f=1 \times 10^{-11} \mathrm{~s}$. A typical value for the exponent is $b=4$.

\section{Boundary/Initial Conditions}

To model a metallic backing behind the foam, we use reflecting (Dirichlet) boundary conditions

$$
[E]_{x=0.1}=0 .
$$

In order to have a finite computational domain, we impose first order absorbing boundary conditions at $x=0$, which are modeled as

$$
\frac{\partial E}{\partial t}-\left.c(\vec{x}) \frac{\partial E}{\partial x}\right|_{x=0}=0 .
$$

With these boundary conditions, ideally a normally incident signal passes out of the computational domain, and does not return, i.e., we force it to be absorbed by the boundary. Note that for signals that are incident at an angle, some reflection occurs. Lastly, to allow for propagation along the top and bottom boundaries ( $y=0$ and $y=0.2$ ), we use insulating boundary conditions

$$
\left[\frac{\partial E}{\partial y}\right]_{y=0}=0, \quad\left[\frac{\partial E}{\partial y}\right]_{y=0.2}=0 .
$$

Also we assume zero initial conditions, i.e.,

$$
E(0, \vec{x})=0, \quad \dot{E}(0, \vec{x})=0 .
$$

\section{Modeling Knit Lines}

The speed of propagation in the domain is given by

$$
c(\vec{x})=\frac{c_{0}}{n(\vec{x})}=\sqrt{\frac{1}{\epsilon(\vec{x}) \mu_{0}}},
$$


where $c_{0}$ is the speed in a vacuum and $n$ is the index of refraction. We model knit lines by changing the index of refraction, thus effectively the speed, in that region. In order to model the speed of wave propagation in the knit lines versus the material surrounding them, we need to be able to distinguish between the respective indices of refraction, i.e., $n_{1}$ in the low density region, and $n_{2}$ in the higher density knit line. Further, we can currently only measure the effective index of refraction of the composite material, $n_{e}$, which is done by computing the "time of flight" in experiments. Thus we need to relate these three indices to each other in order to have accurate estimates for the propagation speed in each region, to use in the simulations.

Experiments have suggested, via time-of-flight measurements, a value for the effective index of refraction as $n_{e}=1.03225 \pm 0.001$. To estimate the index of refraction for foam in the absence of knit lines, blocks of $5 \mathrm{~cm} \times 5 \mathrm{~cm}$ blocks of foam with varying numbers of knit lines layers (between 3 and 7, approximated to the nearest .25) were produced and interrogated. By calculating the velocity of the pulse in these blocks we may extrapolate the data to the case of zero knit line layers. Figure 2 displays the data collected in the experiment.

A line of best fit, computed using linear regression, is plotted in Figure 2 with error bars of two standard deviations. The range of values corresponding to zero knit lines is the estimate for the velocity of the pulse in a region of foam without knit lines. The mean value in this region is $2.94638 \times 10^{8} \mathrm{~m} / \mathrm{s}$ resulting in an index of refraction for the low density region of $n_{1}=1.0172$. Using $n_{e}=(1-\nu) n_{1}+\nu n_{2}$, where $\nu$ is the volume fraction of the knit line region in the foam block. The volume fraction $\nu$ can be estimated by noting the thickness of each knit line divided by the period in which the knit lines occur. Assuming $0.5 \mathrm{~mm}$ knit lines in each $1 \mathrm{~cm}$ of foam corresponds to $\nu=.05$. This gives the index in the knit line as $n_{2}=1.1869$.

In [3], the effective index of refraction $n_{e}$ was approximated via the classical Claussius-Mossotti equation (for instance, see [7] and [8]), by assuming the total polarizability is the sum of the two polarizabilities in each part of the material. The index of refraction in each part of the foam was esti- 


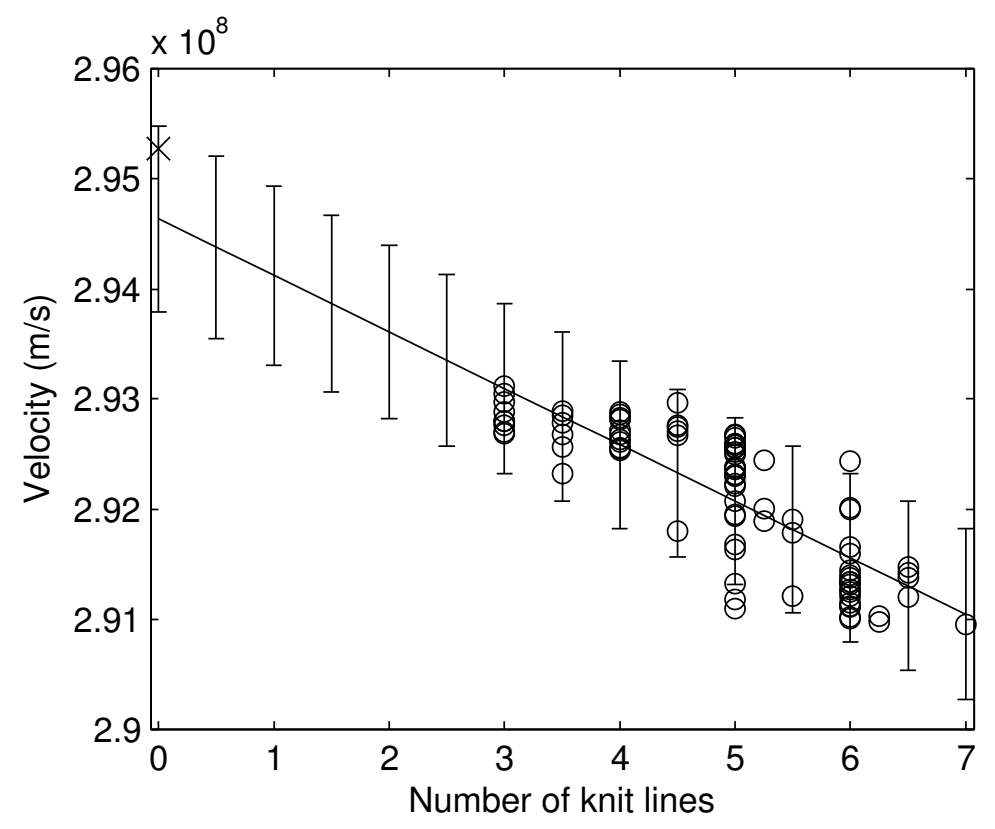

FIGURE 2: Experimental data for the velocity of a THz pulse versus the number of knit lines, with the line of best fit from applying linear regression. The error bars represent two standard deviations; the " $\mathrm{X}$ " denotes the Claussius-Mossotti estimate.

mated to be

$$
\begin{aligned}
& n_{1}=1.01398 \\
& n_{2}=1.03507 .
\end{aligned}
$$

This value is plotted as an " $\mathrm{X}$ " in Figure 2, and is within the range of the error bars of the linear regression estimate.

Although the experimental result agrees well with the theoretical estimate, it is clear from the scattered nature of the data points in Figure 2 that the correlation between the number of knit lines and the velocity is not perfectly linear. The plot in Figure 3 shows that this is mainly due to the rather imprecise relationship between the density and the number of knit lines. The symbol in the plot denotes the frequency (number of occurrences) of data point. The solid line again represents a line of best fit. Only one measurement of the density is beyond two standard deviations from the mean, but many are outside of one standard deviation. Better techniques in estimating the number of knit lines in a block of foam may result in density data more closely linearly related to the number of knit lines, and therefore a more precise estimate of the density for foam in the limit of zero knit lines. 


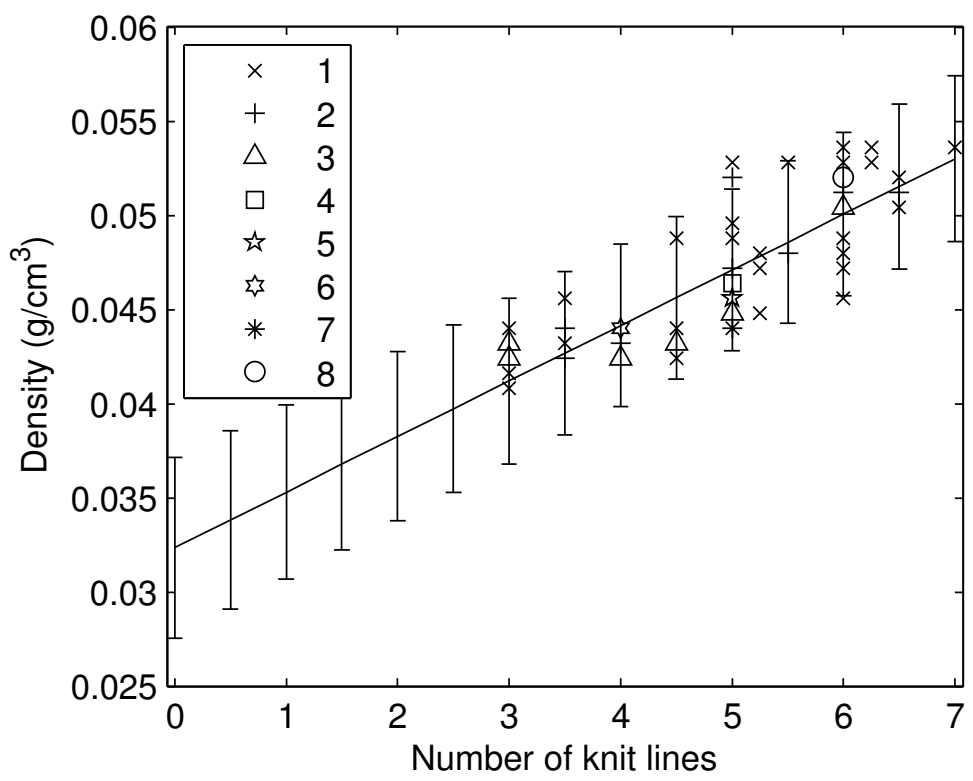

FIGURE 3: Experimental data for the density of a $5 \mathrm{~cm} \times 5 \mathrm{~cm}$ block versus the number of knit lines, with the line of best fit from applying linear regression. The error bars represent two standard deviations; the symbol denotes the frequency (occurrence) of each data point present.

\section{NUMERICAL METHOD}

We employ a (second order) Finite Element method using standard linear two dimensional $(Q 1)$ basis elements to spatially discretize the model described by (1). This results in nine-banded mass and stiffness matrices, $M$ and $S$. We also have a contribution from absorbing boundaries which we denote with $B$. Thus our semi-discrete system for the vector of electric field values $e$ is

$$
M \ddot{e}+B \dot{e}+S e=f .
$$

Here we have absorbed coefficients $\frac{1}{c^{2}}$ and $\frac{1}{c}$ into the definitions of $M$ and $B$, respectively.

For the time derivatives we use second order discretizations (centered differences) for both the first and second derivatives. After collecting all terms involving the updated time step into the left hand side of the equation 
we have the following linear system

$$
A e_{n+1}=b,
$$

where $A$ contains multiples of $M$ and $B$, and $b$ depends on $e_{n}$ and $e_{n-1}$, as well as $S$ and $f$.

As discussed in [3], a mass-lumping approach, using a quadrature rule applied to the basis functions, is most efficient for solving the linear system (5) on each time step considering the length of time span required for propagation problems of this type. LU factorization is limited by memory constraints for large problems, and iterative methods are restricted by computational time.

\section{SIMULATIONS}

We compute numerical simulations of an electromagnetic wave propagating through a material described by its index of refraction, which determines the speed of propagation. We consider the presence of a void similar to what is seen in SOFI when a layer does not completely fill a recess formed in the previous layer. As the foam cures, an elliptical pocket of air is formed. The void is modeled by taking its index of refraction to be that of free space, i.e., $n_{0}=1$. The knit lines are modeled with index of refraction, $n_{2}$, and the surrounding low density regions are described by $n_{1}$. Throughout the following, the knit lines are described using the values estimated above by the use of experimental data.

\section{Plane Wave}

Figure 4 depicts snapshots in time of the propagation of a plane wave incident on a void in the material. The cross denotes the location of the receiver to collect data. The reflection from the void is clearly seen in the third frame. This reflection expands out to form an oblong elliptical wave which eventually returns to the receiver where the signal is recorded.

As the actual transceiver in the experimental setup is devised of a transmitter and receiver which do not occupy the same space (see Figure 5), the direction of pulse propagation is at some non-normal angle to the plane of the foam and the metallic backing. The simulations of the scenario where 

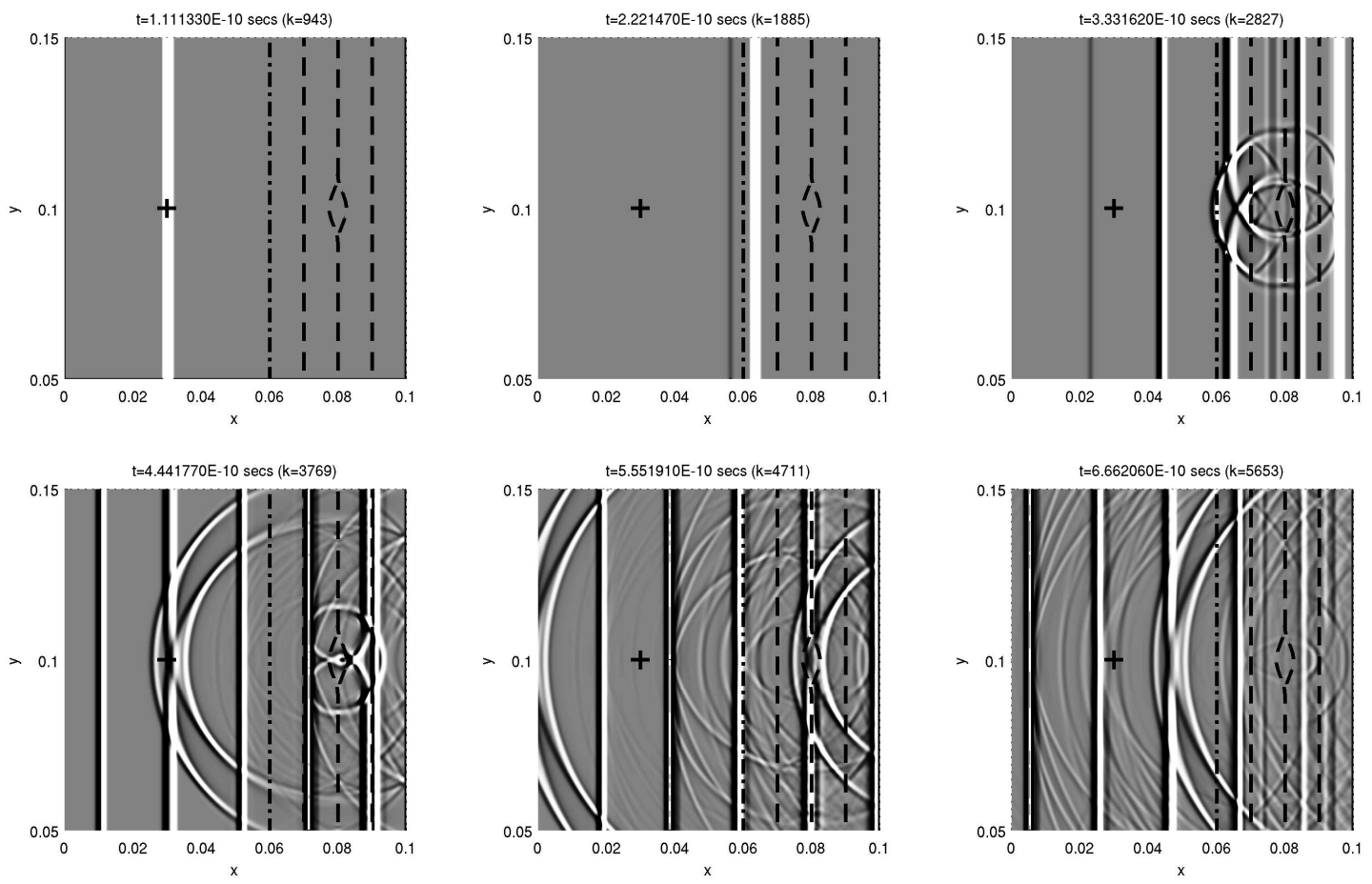

FIGURE 4: Surface plots of solutions for the case where the plane wave pulse is normally incident on the material.

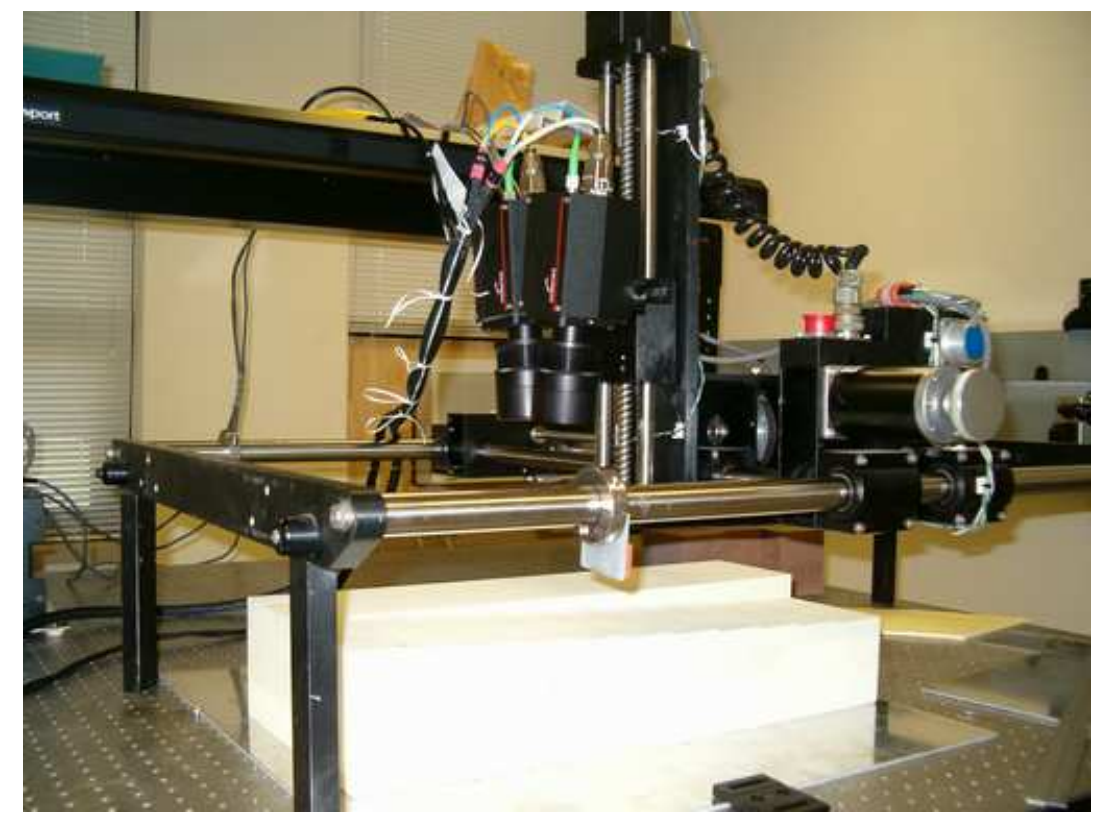

FIGURE 5: Picometrix T-Ray setup used in the electromagnetic interrogation of foam. Note the non-normal incidence and ability to focus. 
the plane wave pulse is incident on the material at a non-normal angle are displayed in Figure 6. While the receiver still located at $x=0.03$, it has been raised in the $y$ direction to collect the center of plane wave reflection.
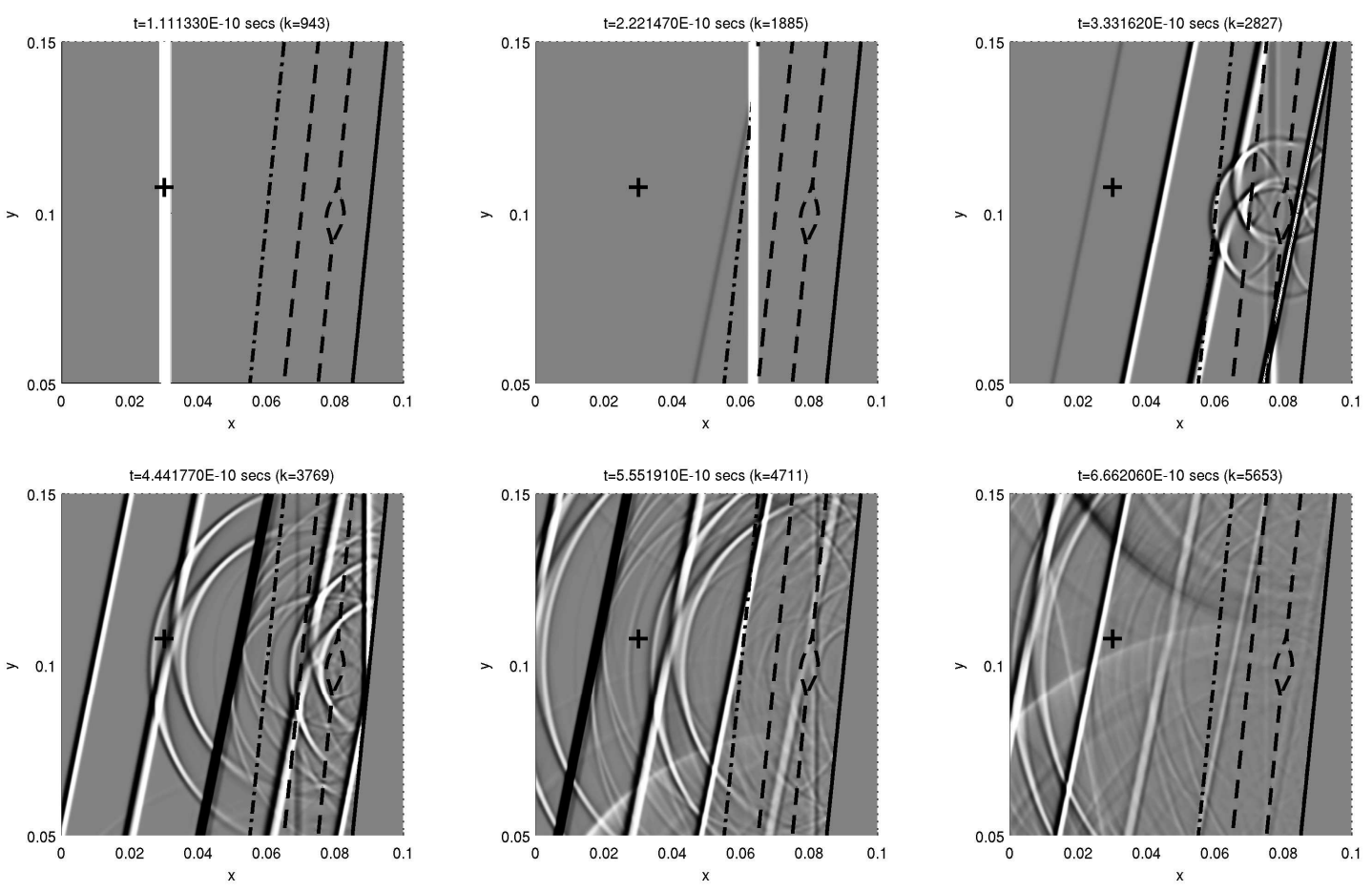

FIGURE 6: Surface plots of solutions for the case where the plane wave pulse is nonnormally incident on the material.

The plots in Figure 7 display the simulated data collected at the receiver for normal and non-normal angles of incidence. In the plot the relative magnitude of the reflection versus the interrogating signal is apparent. The inset displays a plot of the magnification of the reflection from the void. There is a not a significant difference in the two reflections, only a slight timing discrepancy. Note that nearly all of original signal returns even with an oblique angle of incidence.

\section{Focused Wave}

For a focused source we model the interrogating signal using scattered field formulation of point source reflected from elliptical mirror. Note that the top and bottom boundary conditions are now absorbing as we no longer have a plane wave propagating along these boundaries. 

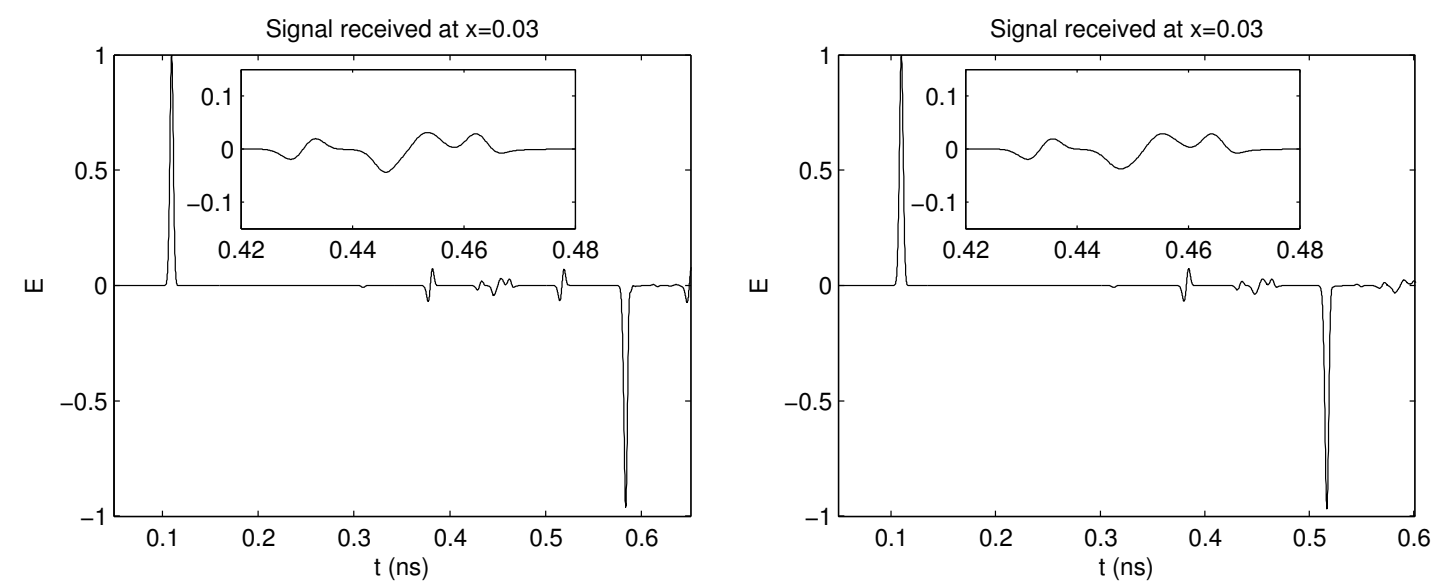

FIGURE 7: The left (right) plot displays the signal received at $x=0$ on center line for the normally (non-normally) incident plane wave case (the inset plot is a magnification of the reflection from the void).

Figure 8 displays snapshots in time of the simulated propagation of a focused wave incident on a void in the material. The reflection from the void is clearly seen in the second frame. This reflection expands out to form an oblong elliptical wave which eventually returns to the antenna where the signal is recorded with a receiver.

The simulations of the scenario where the focused wave pulse is incident on the material at a non-normal angle are displayed in Figure 9. The source is modeled using scattered field formulation of point source reflected from elliptical mirror. The receiver is located at $x=0.03$, but raised to collect center of the focused wave reflection.

The plots in Figure 10 give the simulated data collected at the receiver for the focused wave incident at the two angles of incidence, respectively. The inset again displays a magnified plot of the reflection from the void.

There is a distinct difference in the structure of the two reflections. In particular, the front part of the void reflection is clearly more pronounced in the normally incident case. This suggests that simulations which do not take the proper angle of incidence into account may grossly over-exaggerate the magnitude of the reflection from the void. Note that the amplitude of the void reflections in both cases is larger than that of the plane wave scenario. Further, the difference between the reflection from the beginning of the void is more distinct from the reflection off the back of the void for the focused wave. As the energy from the interrogating wave is concentrated on the 

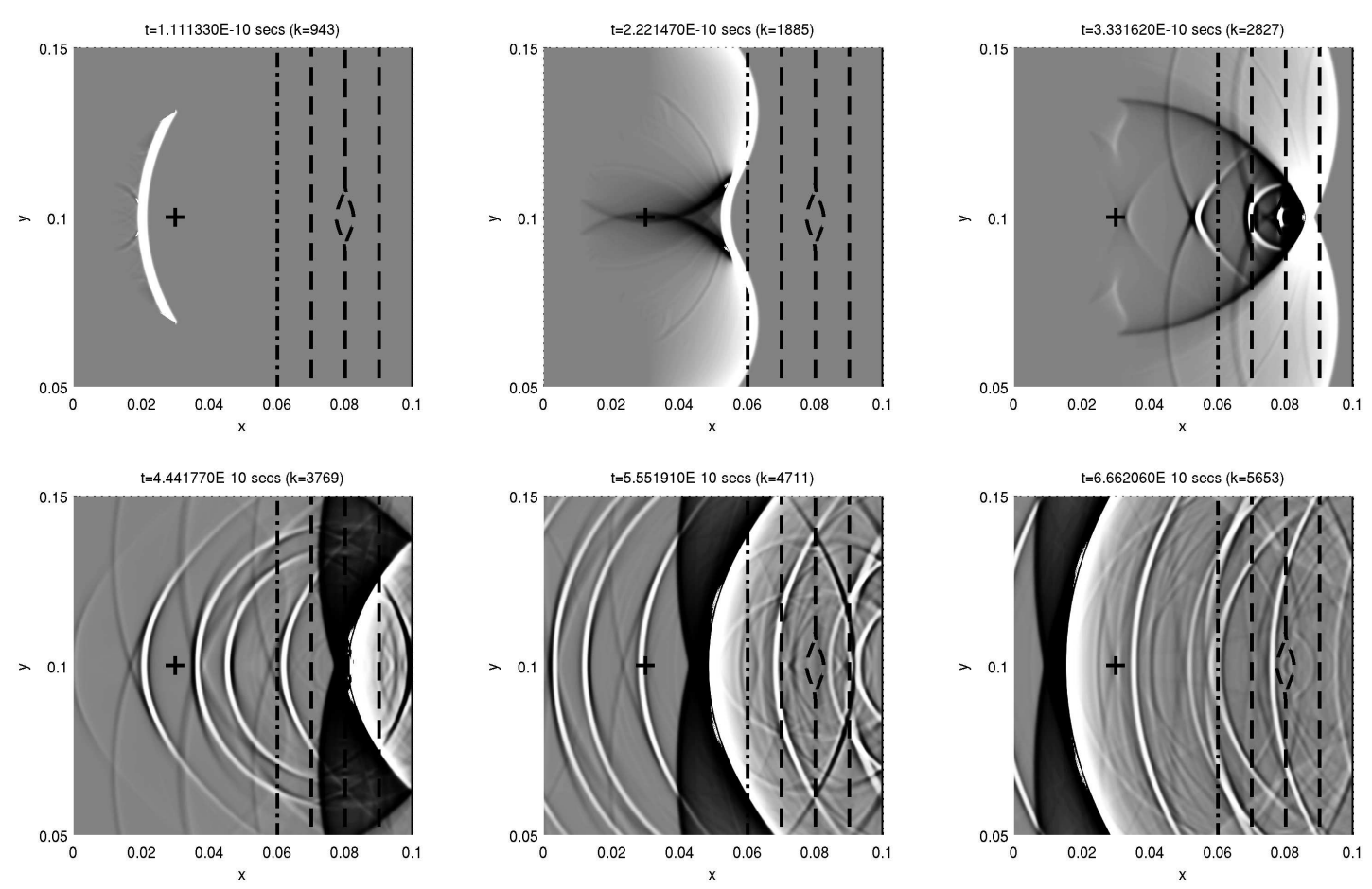

FIGURE 8: Surface plots of solutions for the case where the focused wave pulse is normally incident on the material.

void area, and thus reflected, more information is returned to be collected by the receiver. Conversely, although reflections off the void are larger, the total energy that returns is less than the plane wave simulation. While these facts about focusing are not new results, it is important that any model of a focused pulse validate these basic principles.

\section{CONCLUSIONS}

We have developed a framework which accounts for the presence of knit lines in modeling the electromagnetic propagation of interrogating pulses in SOFI. We were able to compute, using results from a laboratory experiment, estimates for the index of refraction in both the knit lines and the surrounding low-density foam based on measured values from time-of-flight experiments and observable material properties. The values were shown to be consistent with classical physics-based theory assuming constant polarizibility.

In the effort to detect flaws in low-density materials such as foam, highly 

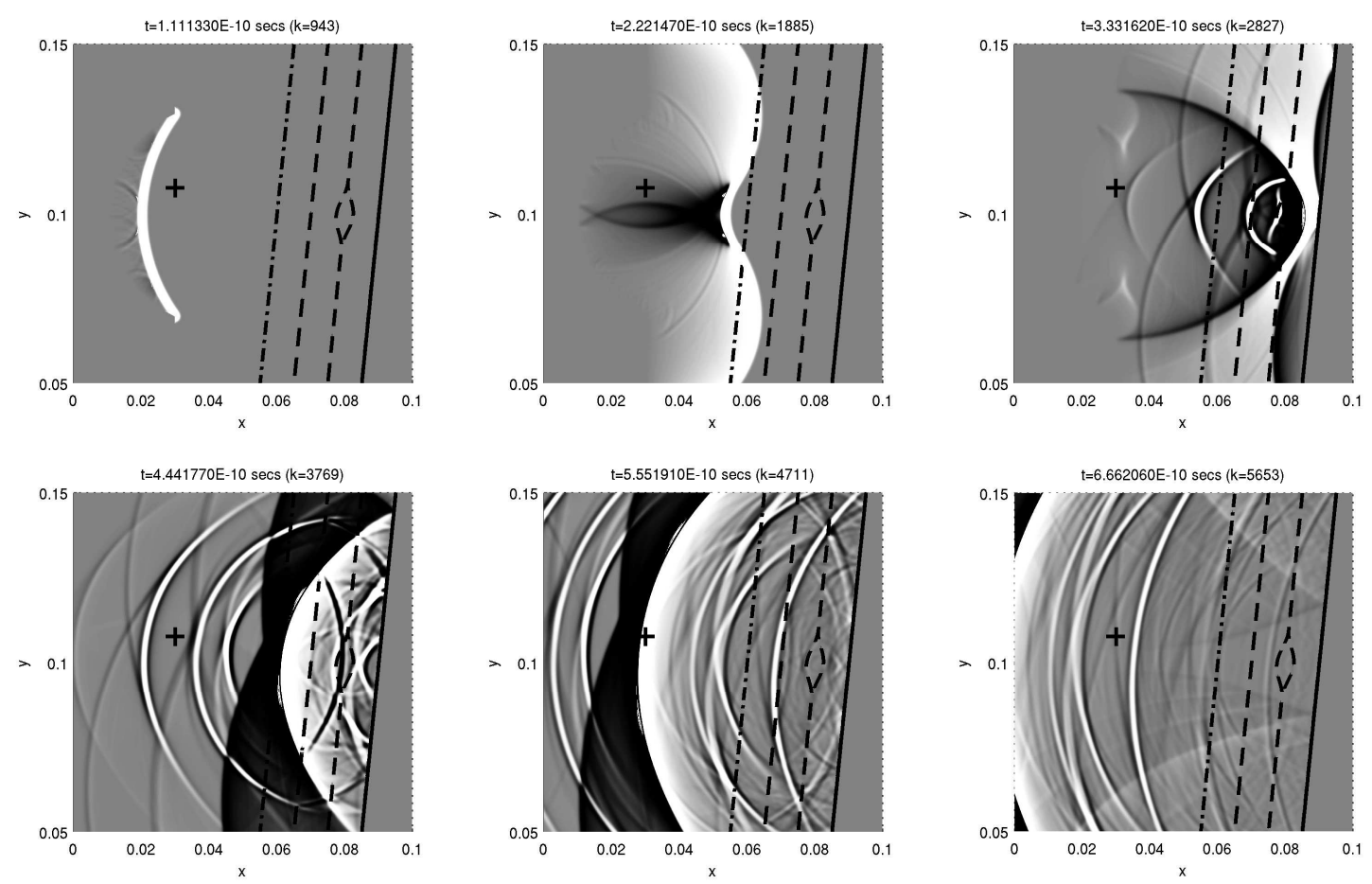

FIGURE 9: Surface plots of solutions for the case where the focused wave pulse is nonnormally incident on the material.
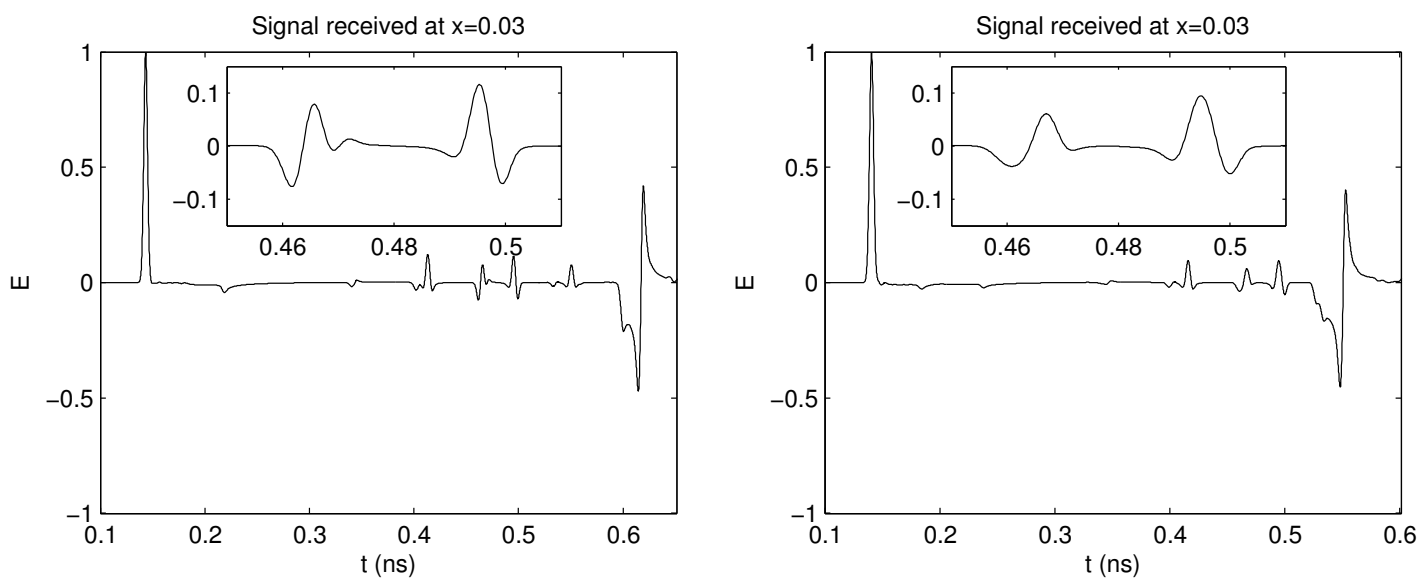

FIGURE 10: The left (right) plot displays the signal received at $x=0$ on center line for the normally (non-normally) incident focused case. The inset plot is a magnification of the reflection from the void.

accurate models must exist to give simulations the precision necessary to distinguish small amplitude reflections from noise, including from model error. While the current formulation may be used as a forward solution in 
an inverse problem context, it is likely that the highest value will lie in its ability to generate synthetic data with which to test faster signal processing approaches to damage detection. Thus it can be used either to explore which shapes of voids are the hardest to detect, or to generate data for scenarios that are difficult or expensive to manufacture.

Our efforts here provide an approach to enhance the accuracy of a model by making it more representative of the material in question and the signal used to interrogate that material. The results themselves suggest that accurate modeling of the angle of incidence is important to quantify the amplitude of the reflected signal, while modeling the focusing captures the clear distinction between the reflections from the front versus the back of the void.

Sufficient experimental results for comparison are difficult to obtain since the amplitude of reflections off of low density materials is very low to immeasurable using currently available power sources at the $\mathrm{THz}$ frequency. Therefore, the results of this paper should be considered as motivation for the development of high power THz devices. For now, experimental information is collected from the aluminum reflection. More work needs to be done to match simulations to this data, including adding attenuation, possibly via coupling Maxwell's equations to a time domain model of a scattering mechanism.

\section{ACKNOWLEDGEMENTS}

This research was supported in part by the U.S. Air Force Office of Scientific Research under grant AFOSR FA9550-04-1-0220 and in part by the National Institute of Aerospace (NIA) and NASA under grant NIA/NCSU03-01-2536-NC.

\section{REFERENCES}

[1] J. Xu, et. al., T-rays identify defects in insulating materials, Conference of Laser and Electro-Optics (CLEO) 2004, San Francisco, CA, May 2004. 
[2] G. Zhao, M. Mors, T. Wenckebach, and P. Planken, Terahertz dielectric properties of polystyrene foam, J. Opt. Soc. Amer. B, vol. 19, pp. 14761476, 2002.

[3] H. T. Banks and N. L. Gibson, "Void detection in foam with knit lines using THz pulse interrogation", Tech. Rep. CRSC-TR06-05, Center for Research in Scientific Computation, North Carolina State University, February 2006. To appear in Mathematical and Computer Modelling.

[4] H. Zhong, J. Xu, X. Xie, T. Yuan, R. Reightler, E. Madaras, and X.C. Zhang, Nondestructive defect identification with terahertz time-offlight tomography, IEEE Sensors Journal, vol. 5, 2, pp. 203-208, 2005.

[5] H. T. Banks, N. L. Gibson and W. P. Winfree, "Gap detection with electromagnetic terahertz signals", Nonlinear Analysis: Real World Applications 6, 381-416, 2005. Tech. Rep. CRSC-TR03-40, Center for Research in Scientific Computation, North Carolina State University, September 2003.

[6] H. T. Banks and N. L. Gibson, "Inverse Problems Involving Maxwell's Equations with a Distribution of Dielectric Parameters", Tech. Rep. CRSC-TR05-29, Center for Research in Scientific Computation, North Carolina State University, July 2005. To appear in the Quarterly of Applied Mathematics.

[7] P. Wang, A. Beck, W. K orner, H. Scheller, and J. Fricke, "Density and refractive index of silica aerogels after low- and high-temperature supercritical drying and thermal treatment", J. Phys. D: Appl. Phys. 27, pp. 414-418, 1994.

[8] F. S. Solheim, J. Vivekanandan, R. H. Ware, and C. Rocken, "Propogation delays induced in GPS signals by dry air, water vapor, hydrometeors, and other particulates", J. Geophys. Res., vol. 104, D8, pp. 9663-9670, 1999. 